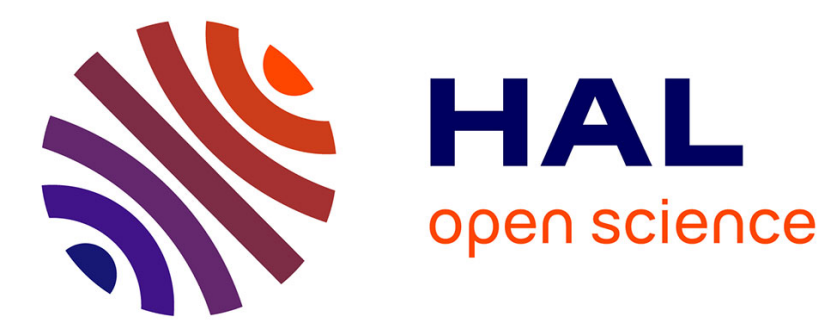

\title{
Real-time estimation for switched linear systems
}

Michel Fliess, Cédric Join, Wilfrid Perruquetti

\section{To cite this version:}

Michel Fliess, Cédric Join, Wilfrid Perruquetti. Real-time estimation for switched linear systems. 47th IEEE Conference on Decision and Control, Dec 2008, Cancun, Mexico. inria-00318499

\section{HAL Id: inria-00318499 \\ https://hal.inria.fr/inria-00318499}

Submitted on 4 Sep 2008

HAL is a multi-disciplinary open access archive for the deposit and dissemination of scientific research documents, whether they are published or not. The documents may come from teaching and research institutions in France or abroad, or from public or private research centers.
L'archive ouverte pluridisciplinaire HAL, est destinée au dépôt et à la diffusion de documents scientifiques de niveau recherche, publiés ou non, émanant des établissements d'enseignement et de recherche français ou étrangers, des laboratoires publics ou privés. 


\title{
Real-time estimation for switched linear systems
}

\author{
Michel FLIESS, Cédric JOIN, Wilfrid PERRUQUETTI
}

\begin{abstract}
We extend previous works on real-time estimation, via algebraic techniques, to the recovering of the switching signal and of the state for switching linear systems. We characterize also singular inputs for which the switched systems become undistinguishable. Several convincing numerical experiments are illustrating our techniques which are easily implementable.
\end{abstract}

Keyword-Linear systems, switching systems, hybrid systems, state estimation, switching signal estimation, numerical differentiation.

\section{INTRODUCTION}

Many systems encountered in practice exhibit switchings between several subsystems, both as a result of controller design, such as in switching supervisory control, and inherently by nature, such as when a physical plant has the capability of undergoing several operational modes.

Switched systems may be viewed as higher-level abstractions of hybrid systems, obtained by neglecting the details of the discrete behavior. Informally, a switched system is composed of a family of dynamical subsystems (linear or nonlinear), and a rule, called the switching law, that orchestrates the switching between them. In recent years, there has been increasing interest in the control problems of switched systems due to their significance from both a theoretical and practical point of view and also because of their inherently interdisciplinary nature. So several important results for switched systems have been achieved, including various results on stability [1], [3], [5], [9], [10], [18], [20], [36], [21], [28], [31] (with many applications see, for example, [12] for application to electrical power converters), stabilization [6], [23], [25], [26], [27], [39], [38], [40], [42], [37], tracking [7], controllability results [32], [41], and inputto-state properties, .... See, e.g., [8], [11], [20], [19], [33] for a survey of this type of results.

Observability and state estimation is a key problem for such systems, where discrete and continuous parts are mixed. Consider, for instance, a finite set of ordinary differential equations (ODE) ${ }^{1} \dot{x}=f_{i}(t, x, u)$, where $f_{i}: \mathbb{R} \times \mathbb{R}^{n} \times \mathbb{R}^{m} \rightarrow$ $\mathbb{R}^{n}, \quad i \in I \triangleq\{1, \ldots, M\}$. The vector-valued output is a

Michel FLIESS is with INRIA-ALIEN \& LIX (UMR-CNRS 7161), École polytechnique, 91128 Palaiseau, France. E-mail: Michel.Fliess@polytechnique.edu

Cédric JOIN is with INRIA-ALIEN \& CRAN (UMR-CNRS 7039), Nancy-Université, BP 239, 54506 Vandœuvre-lès-Nancy, France. E-mail: Cedric.Join@cran.uhp-nancy. fr

Wilfrid PERRUQUETTI is with INRIA-ALIEN \& École Centrale de Lille, LAGIS (UMR-CNRS 8146), BP 48, Cité Scientifique, 59651 Villeneuve-d'Ascq, France. E-mail: wilfrid.perruquetti@ec-lille.fr.

${ }^{1}$ In the rest of the paper the time argument is omitted for sake of brevity when the context is clear for example $f_{i}(t, x, u)$ should be read as $f_{i}(t, x(t), u(t))$. function of the continuous state. The observation problem is to determine the continuous state and the active ODE driving the current continuous state evolution. A generic setting for the observability of switched linear systems in continuous setting has been given in [4]. In [2], [34], [35] the observability of switched linear systems in the case of deterministic switching signal was carried out. Unobserved switching case was analyzed in [29].

It is clear that if one knows which current ODE is active (the current index " $i$ ") then one can say something about the current continuous state. Thus a key problem is the real time computation of the so-called switching signal defined by $\sigma(t, x): \mathbb{R} \times \mathbb{R}^{n} \rightarrow I,(t, x) \mapsto \sigma(t, x)$, where $\sigma(t, x) \in$ $I \triangleq\{1, \ldots, M\}$ corresponds to the index associated with the current active ODE. In the rest of the paper we will only consider linear ordinary differential equations (LODE). Taking a pair of such LODE, one can distinguish the two LODE if for any non trivial input the two systems produce two different outputs (roughly speaking). On this basis, one can encounter the following facts:

1) some pairs of LODE may be indistinguishable: any non trivial input will produce for the two systems the same output.

2) for a given pair of LODE, some peculiar inputs will produce the same outputs: the two systems are indistinguishable. For example, for the two following LODE $\dot{y}_{1}=u_{1}, \ddot{y}_{2}+\dot{y}_{2}=2 u_{2}$, it is clear that if the input of these systems is $u_{1}=u_{2}=\exp (t)$ then the two systems have a common trajectory namely $y(t)=\exp (t)$

It is thus important to characterize such singular inputs and then to give efficient tools for observations.Except for these pathological cases, the state (continuous and discrete or the switching signal) reconstruction of an hybrid system is a three stage process as follows:

1) using the measured output $y$ (and eventually the input $u$ ) one needs to reconstruct $y, \dot{y}, \ldots, y^{\left(k_{y}\right)}$ up to some finite order $k_{y}$ which is not necessarily equal to the state dimension ( $k_{y}$ may be smaller) and eventually $u, \dot{u}, \ldots, u^{\left(k_{m}\right)}$,

2) reconstruct the switching signal or the discrete state: a set of signal are constructed using the obtained reconstructed signals $y, \dot{y}, \ldots, y^{\left(k_{y}\right)} ; u, \dot{u}, \ldots, u^{\left(k_{m}\right)}$ in order to distinguish the active subsystem.

3) reconstruct the continuous state: using for example step 1 or eventually a refined technic using the knowledge of the actual active subsystem. 


\section{Dinstinguishability}

\section{A. Problem formulation: an Input-output behavior}

Consider switched monovariable ${ }^{2}$ linear systems of the form:

$$
\begin{aligned}
& \dot{x}=A_{\sigma(t)} x+B_{\sigma(t)} u, \\
& y=C_{\sigma(t)} x+D_{\sigma(t)} u, \\
& x \in \mathbb{R}^{n}, u \in \mathbb{R}, y \in \mathbb{R},
\end{aligned}
$$

where $\sigma(t)$ is the switching signal taking value within the index set $I=\{1, \ldots, M\}$. In the rest we address the problem of the reconstruction of the switching signal $\sigma(t)$ in "realtime", and of the state variables if is possible. From the problem formulation it is clear that for each $i \in I$ we only need an input/output representation of our linear subsystems. In the rest we adopt a matrix transfer representation of the input/output behavior:

$$
\mathfrak{a}_{i}\left(\frac{d}{d t}\right) y_{i}=\mathfrak{b}_{i}\left(\frac{d}{d t}\right) u, \quad i \in I .
$$

\section{B. Distinguishability}

Consider two monovariable linear systems with transfer functions $\frac{\mathfrak{b}_{i}}{\mathfrak{a}_{i}}, i=1,2, \mathfrak{a}_{i}, \mathfrak{b}_{i} \in \mathbb{R}[s], \mathfrak{b}_{i} \neq 0,\left(\mathfrak{a}_{i}, \mathfrak{b}_{i}\right)=1$. It is clear that they do not exhibit the same input-output behavior if the two transfer functions are different, i.e., $\mathfrak{a}_{1} \mathfrak{b}_{2}-\mathfrak{a}_{2} \mathfrak{b}_{1} \neq$ 0 . There might exist nevertheless particular inputs for which the two outputs coincide.

Assume that two input-output systems satisfy $\mathfrak{a}_{i}\left(\frac{d}{d t}\right) y_{i}=$ $\mathfrak{b}_{i}\left(\frac{d}{d t}\right) u, i=1,2$. It is clear that we cannot distinguish their input-output behaviors if, and only if, $u$ and $y=y_{1}=y_{2}$ satisfy the matrix differential equation

$$
\left(\begin{array}{ll}
\mathfrak{a}_{1} & -\mathfrak{b}_{1} \\
\mathfrak{a}_{2} & -\mathfrak{b}_{2}
\end{array}\right)\left(\begin{array}{l}
y \\
u
\end{array}\right)=\left(\begin{array}{l}
0 \\
0
\end{array}\right) .
$$

Classic algebraic manipulations show that Eq. (3) is equivalent to

$$
\mathfrak{A}\left(\frac{d}{d t}\right) u=0 \mathfrak{B}\left(\frac{d}{d t}\right) y=0 .
$$

where $\mathfrak{A}, \mathfrak{B} \in \mathbb{R}\left[\frac{d}{d t}\right], \mathfrak{A} \mathfrak{B} \neq 0$. The two systems are said to be strongly distinguishable if, and only if, $\mathfrak{A}$ and $\mathfrak{B}$ are constant. This is equivalent saying that the two systems have the same input-output behavior only for $u=y_{1}=y_{2}=0$. If not the two systems are said to be weakly distinguishable. The next result summarizes the above computations.

Theorem 1 In (4) $\mathfrak{A}$ and $\mathfrak{B}$ are given by

$$
\begin{gathered}
\mathfrak{A}=\operatorname{gcd}\left(\mathfrak{b}_{1} \mathfrak{p}_{1}^{1}, \mathfrak{b}_{2} \mathfrak{p}_{1}^{2}\right), \\
\mathfrak{B}=\left(\mathfrak{a}_{2} \mathfrak{b}_{1}^{\prime}-\mathfrak{a}_{1} \mathfrak{b}_{2}^{\prime}\right),
\end{gathered}
$$

where $\mathfrak{b}=\operatorname{gcd}\left(\mathfrak{b}_{1}, \mathfrak{b}_{2}\right), \mathfrak{b}_{1}=\mathfrak{b} \mathfrak{b}_{1}^{\prime}, \mathfrak{b}_{2}=\mathfrak{b} \mathfrak{b}_{2}^{\prime}, \mathfrak{a}=$ $\operatorname{gcd}\left(\mathfrak{a}_{1}, \mathfrak{a}_{2}\right), \mathfrak{a}_{1}=\mathfrak{a} \mathfrak{a}_{1}^{\prime}, \mathfrak{a}_{2}=\mathfrak{a} \mathfrak{a}_{2}^{\prime}, \mathfrak{p}^{1}=\operatorname{lcm}\left(\mathfrak{a}_{1}^{\prime}, \mathfrak{a}_{2}^{\prime} \mathfrak{b}_{1}^{\prime}-\mathfrak{a}_{1}^{\prime} \mathfrak{b}_{2}^{\prime}\right)$, and $\mathfrak{p}^{1}=\mathfrak{p}_{1}^{1} \mathfrak{a}_{1}^{\prime}=\mathfrak{p}_{2}^{1}\left(\mathfrak{a}_{2}^{\prime} \mathfrak{b}_{1}^{\prime}-\mathfrak{a}_{1}^{\prime} \mathfrak{b}_{2}^{\prime}\right), \mathfrak{p}^{2}=\operatorname{lcm}\left(\mathfrak{a}_{2}^{\prime}, \mathfrak{a}_{2}^{\prime} \mathfrak{b}_{1}^{\prime}-\right.$ $\left.\mathfrak{a}_{1}^{\prime} \mathfrak{b}_{2}^{\prime}\right)$, and $\mathfrak{p}^{2}=\mathfrak{p}_{1}^{2} \mathfrak{a}_{2}^{\prime}=\mathfrak{p}_{2}^{2}\left(\mathfrak{a}_{2}^{\prime} \mathfrak{b}_{1}^{\prime}-\mathfrak{a}_{1}^{\prime} \mathfrak{b}_{2}^{\prime}\right)$. Strong distinguishability is equivalent to $\left(\mathfrak{a}_{2} \mathfrak{b}_{1}^{\prime}-\mathfrak{a}_{1} \mathfrak{b}_{2}^{\prime}\right) \in \mathbb{R} \backslash\{0\}$ and $\operatorname{gcd}\left(\mathfrak{b}_{1} \mathfrak{p}_{1}^{1}, \mathfrak{b}_{2} \mathfrak{p}_{1}^{2}\right) \in \mathbb{R} \backslash\{0\}$.

\footnotetext{
${ }^{2}$ Extension to the multivariable case will be discussed in a coming paper.
}

Proof: Omitted for sake of brevity (Just use simple algebraic manipulations on polynomials).

Remark 2 The two systems are said to be strongly distinguishable if, and only if, the only solution to (4)-(5) is the trivial one that is $y=u=0$. This is equivalent saying that the two systems have the same input-output behavior only for $u=y_{1}=y_{2}=0$. If not the two systems are said to be weakly distinguishable.

\section{Example: first order systems}

Let us consider (1) with $n=1$ and $M=2$ : for a given $i \in I$ the corresponding system has a transfert function of the form

$$
F_{i}(s)=\frac{k_{i}}{1+\tau_{i} s}, \quad k_{i}=\frac{b_{i}}{a_{i}}, \tau_{i}=\frac{1}{a_{i}},
$$

the two transfer functions are different iff

$$
k_{1}\left(1+\tau_{2} s\right)-k_{2}\left(1+\tau_{1} s\right) \neq 0 .
$$

Nevertheless for the two systems some input/output behavior cannot be distinguish iff

$$
\left(\begin{array}{cc}
\left(1+\tau_{1} s\right) & -k_{1} \\
\left(1+\tau_{2} s\right) & -k_{2}
\end{array}\right)\left(\begin{array}{l}
y \\
u
\end{array}\right)=\left(\begin{array}{l}
0 \\
0
\end{array}\right) .
$$

a) $k_{1} \tau_{2}=k_{2} \tau_{1}$ and $k_{1} \neq k_{2}$, thus (8) has a unique singular behavior for which the two systems can not be distinguish $u=0, y=0$, which means that, when at rest, the two systems can not be distinguished: they are strongly distinguishable. This result comes directly from Theorem 1: since $\mathfrak{a}_{1}=1+\tau_{1} s, \mathfrak{b}_{1}=k_{1}, \mathfrak{a}_{2}=1+\tau_{2} s, \mathfrak{b}_{2}=k_{2}$ we have $\left(\mathfrak{a}_{2}^{\prime} \mathfrak{b}_{1}^{\prime}-\mathfrak{a}_{1}^{\prime} \mathfrak{b}_{2}^{\prime}\right)=k_{1}-k_{2}$, it comes out that $\mathfrak{p}^{1}=\operatorname{lcm}\left(\mathfrak{a}_{1}^{\prime}, \mathfrak{a}_{2}^{\prime} \mathfrak{b}_{1}^{\prime}-\mathfrak{a}_{1}^{\prime} \mathfrak{b}_{2}^{\prime}\right)=\left(k_{1}-k_{2}\right)\left(1+\tau_{1} s\right), p^{2}=$ $\operatorname{lcm}\left(\mathfrak{a}_{2}^{\prime}, \mathfrak{a}_{2}^{\prime} \mathfrak{b}_{1}^{\prime}-\mathfrak{a}_{1}^{\prime} \mathfrak{b}_{2}^{\prime}\right)=\left(k_{1}-k_{2}\right)\left(1+\tau_{2} s\right)$ and thus Eq (4) and (5) reads as

$$
\begin{gathered}
\operatorname{gcd}\left(\mathfrak{b}_{1} \mathfrak{p}_{1}^{1}, \mathfrak{b}_{2} \mathfrak{p}_{1}^{2}\right) u=0 \Leftrightarrow\left(k_{1}-k_{2}\right) u=0, \\
\left(\mathfrak{a}_{2} \mathfrak{b}_{1}^{\prime}-\mathfrak{a}_{1} \mathfrak{b}_{2}^{\prime}\right) y=0 \Leftrightarrow\left(k_{1}-k_{2}\right) y=0,
\end{gathered}
$$

leading to the following singular i/o: $y=0, u=0$.

b) $k_{1} \tau_{2}-k_{2} \tau_{1} \neq 0$, thus (8) has a unique singular behavior for which the two systems can not be distinguish

$$
\begin{aligned}
& 0=\left(k_{1} \tau_{2}-k_{2} \tau_{1}\right) \dot{y}+\left(k_{1}-k_{2}\right) y, \\
& u=\frac{\tau_{1} \dot{y}+y}{k_{1}}=\frac{\tau_{2} \dot{y}+y}{k_{2}},
\end{aligned}
$$

solutions of (10) are of the form $y=c \exp (\alpha t), \alpha=$ $\frac{-\left(k_{1}-k_{2}\right)}{\left(k_{1} \tau_{2}-k_{2} \tau_{1}\right)}$ which include the case $c$ (constant) when $k_{1}=$ $k_{2}$; moreover (11) gives $u=c \frac{\tau_{1} \alpha+1}{k_{1}} \exp (\alpha t)$ leading to $u=d \exp (\alpha t)$ with $d=c \frac{\left(\tau_{2}-\tau_{1}\right)}{\left(k_{1} \tau_{2}-k_{2} \tau_{1}\right)}$. Thus (8) has a unique singular behavior for which the two systems can not be distinguish $y=c, u=\frac{c}{k_{1}}$ when $k_{1}=k_{2}$ or $y=c \exp (\alpha t), u=d \exp (\alpha t)$, when $k_{1} \neq k_{2}$. This can be obtained directly suing Theorem 1, for example:

- When $k_{1}=2, \tau_{1}=1, k_{2}=1, \tau_{2}=2: k_{1} \tau_{2}-k_{2} \tau_{1}=$ $3 \neq 0$, one has $\mathfrak{a}_{1}=s+1, \mathfrak{b}_{1}=2, \mathfrak{a}_{2}=2 s+1, \mathfrak{b}_{2}=1$ 
from which one gets $\mathfrak{b}_{1}^{\prime}=2, \mathfrak{b}_{2}^{\prime}=1, \mathfrak{a}_{1}=\mathfrak{a}_{1}^{\prime}, \mathfrak{a}_{2}=\mathfrak{a}_{2}^{\prime}$, and finally :

$$
\left.\left(\mathfrak{a}_{2}^{\prime} \mathfrak{b}_{1}^{\prime}-\mathfrak{a}_{1}^{\prime} \mathfrak{b}_{2}^{\prime}\right)\right)=3 s+1 .
$$

Thus $\mathfrak{p}^{1}=\operatorname{lcm}(s+1,3 s+1)=(s+1)(3 s+1)$ and $\mathfrak{p}^{2}=\operatorname{lcm}(2 s+1,3 s+1)=(2 s+1)(3 s+1)$ leads to

$\mathfrak{p}^{1}=\mathfrak{p}_{1}^{1} \mathfrak{a}_{1}^{\prime}=\mathfrak{p}_{2}^{1}\left(\mathfrak{a}_{2}^{\prime} \mathfrak{b}_{1}^{\prime}-\mathfrak{a}_{1}^{\prime} \mathfrak{b}_{2}^{\prime}\right), \mathfrak{p}_{1}^{1}=(3 s+1), \mathfrak{p}_{2}^{1}=(s+1)$ $\mathfrak{p}^{2}=\mathfrak{p}_{1}^{2} \mathfrak{a}_{2}^{\prime}=\mathfrak{p}_{2}^{2}\left(\mathfrak{a}_{2}^{\prime} \mathfrak{b}_{1}^{\prime}-\mathfrak{a}_{1}^{\prime} \mathfrak{b}_{2}^{\prime}\right), \mathfrak{p}_{1}^{2}=(3 s+1), \mathfrak{p}_{2}^{2}=(2 s+1$

Eq (4) and (5) reads as

$$
\begin{gathered}
\operatorname{gcd}\left(\mathfrak{b}_{1} \mathfrak{p}_{1}^{1}, \mathfrak{b}_{2} \mathfrak{p}_{1}^{2}\right) u=0 \Leftrightarrow(3 s+1) u=0, \\
\left(\mathfrak{a}_{2} \mathfrak{b}_{1}^{\prime}-\mathfrak{a}_{1} \mathfrak{b}_{2}^{\prime}\right) y=0 \Leftrightarrow(3 s+1) y=0,
\end{gathered}
$$

leading to the following singular i/o: $y=y_{0} \exp (-t / 3)$, $u=u_{0} \exp (-t / 3)$, note that $\frac{y_{0}}{3}=u_{0}$.

- When $k_{1}=1, \tau_{1}=1, k_{2}=2, \tau_{2}=1: k_{1} \tau_{2}-k_{2} \tau_{1}=$ $-1 \neq 0$, one has $\mathfrak{a}_{1}=s+1, \mathfrak{b}_{1}=1, \mathfrak{a}_{2}=s+1, \mathfrak{b}_{2}=2$ from which one gets $\mathfrak{b}_{1}^{\prime}=1, \mathfrak{b}_{2}^{\prime}=2, \mathfrak{a}_{1}=\mathfrak{a}_{1}^{\prime}=\mathfrak{a}_{2}=$ $\mathfrak{a}_{2}^{\prime}$, and finally

$$
\left(\mathfrak{a}_{2}^{\prime} \mathfrak{b}_{1}^{\prime}-\mathfrak{a}_{1}^{\prime} \mathfrak{b}_{2}^{\prime}\right)=-(s+1) .
$$

Thus $\mathfrak{p}^{1}=\operatorname{lcm}(s+1, s+1)=(s+1)$ and $\mathfrak{p}^{2}=\operatorname{lcm}(s+$ $1, s+1)=(s+1)$ leads to

$$
\begin{aligned}
& \mathfrak{p}^{1}=\mathfrak{p}_{1}^{1} \mathfrak{a}_{1}^{\prime}=\mathfrak{p}_{2}^{1}\left(\mathfrak{a}_{2}^{\prime} \mathfrak{b}_{1}^{\prime}-\mathfrak{a}_{1}^{\prime} \mathfrak{b}_{2}^{\prime}\right), \mathfrak{p}_{1}^{1}=1, \mathfrak{p}_{2}^{1}=-1, \\
& \mathfrak{p}^{2}=\mathfrak{p}_{1}^{2} \mathfrak{a}_{2}^{\prime}=\mathfrak{p}_{2}^{2}\left(\mathfrak{a}_{2}^{\prime} \mathfrak{b}_{1}^{\prime}-\mathfrak{a}_{1}^{\prime} \mathfrak{b}_{2}^{\prime}\right), \mathfrak{p}_{1}^{2}=1, \mathfrak{p}_{2}^{2}=-1,
\end{aligned}
$$

$\mathrm{Eq}(4)$ and (5) reads as

$$
\begin{gathered}
\operatorname{gcd}\left(\mathfrak{b}_{1} \mathfrak{p}_{1}^{1}, \mathfrak{b}_{2} \mathfrak{p}_{1}^{2}\right) u=0 \Leftrightarrow u=0, \\
\left(\mathfrak{a}_{2} \mathfrak{b}_{1}^{\prime}-\mathfrak{a}_{1} \mathfrak{b}_{2}^{\prime}\right) y=0 \Leftrightarrow(s+1) y=0,
\end{gathered}
$$

leading to the following singular i/o: $u=0, y=$ $y_{0} \exp (-t)$.

\section{SWITCHING SIGNAL ESTIMATION}

Assume from now on that all the subsystems models are known and that any pair is strongly distinguishable. Let us consider a switching system defined by a finite collection of input/output behaviors driven by LODE satisfying the above given assumptions. As soon as the system is not at rest, for the given control, the measured output can be used to determine which subsystem is active. From now we want to obtain effective real-time algorithm to determine the current " $i$ ". If one is able to construct in real time the following quantities

$$
r_{i}(t)=\mathfrak{a}_{i}\left(\frac{d}{d t}\right) y_{i}-\mathfrak{b}_{i}\left(\frac{d}{d t}\right) u,
$$

it is clear that the current " $i$ " is such that $r_{i}(t)=0$ on a sub-set of $\mathbb{R}$ with non zero measure. The problem is thus reduced to the real-time computation of time derivative of the output and input despite the noise.

The numerical differentiation technics introduced below are of non asymptotic nature, and the desired estimation can be obtained instantaneously (there is a singularity at time $t=0$ ). But in practice they are numerically implemented with discrete measured data, thus from a practical point of view, it will be necessary that the sampling time should be small enough with respect to the duration time between two successive switchings ${ }^{3}$.

\section{A. Numerical differentiation}

Consider a signal ${ }^{4} y(t)=\sum_{i=0}^{\infty} y^{(i)}(0) \frac{t^{i}}{i !}$ which is assumed to be analytic around $t=0$ and its truncated Taylor expansion $y_{N}(t)=\sum_{i=0}^{N} y^{(i)}(0) \frac{t^{i}}{i !}$ at order $N$. The usual 1 tules of symbolic calculus in Schwartz's distribution theory [30] yield $y_{N}^{(N+1)}(t)=y(0) \delta^{(N)}+\ldots+y^{(N)}(0) \delta$, where $\delta$ is the Dirac measure at zero. Multiply both sides by $(-t)^{i}$ and apply the rules $t \delta=0, t \delta^{(i)}=-i \delta^{(i-1)}, i \geq 1$. We obtain a triangular system of linear equations from which the derivatives $y^{(i)}(0)$ can be obtained $(1 \leq i \leq N)$

$$
(-t)^{i} y_{N}^{(N+1)}(t)=\frac{N !}{(N-i) !} \delta^{(N-i)} y(0)+\ldots+\delta y^{(N-i)}(0)
$$

It means that the coefficients $y(0), \ldots, y^{(N)}(0)$ are linearly identifiable [16], [17]. The time derivatives of $y_{N}(t)$, the Dirac measures and its derivatives are removed by integrating with respect to time both sides of Eq. (16) at least $\nu$ times $(\nu>N)$ :

$$
\begin{gathered}
\int_{0}^{t} \int_{0}^{t_{\nu-1}} \cdots \int_{0}^{t_{1}}(-\tau)^{i} y_{N}^{(N+1)} d t_{\nu-1} \cdots d t_{1} d \tau= \\
\frac{N !}{(N-i) !} \frac{t^{\nu-N-i-1}}{(\nu-N-i-1) !} y(0)+\ldots+\frac{t^{\nu-1}}{(\nu-1) !} y^{(N-i)}(0)
\end{gathered}
$$

It is clear that the numerical estimation rely on $\lim _{N \rightarrow+\infty}\left[y_{N}^{(i)}(0)\right]_{\text {estim }}(t)=y^{(i)}(0)$.

Remark 3 These iterated integrals are low pass filters which attenuate the noises, which are viewed as highly fluctuating phenomena (see [13] for more details).

Remark 4 The above formulae may easily be extended to sliding time windows in order to obtain real time estimates (see [22] for further details).

\section{B. Algorithm}

Off line:

1) determine the maximum number of output derivative necessary for estimation (take the highest derivative of the output in the collection of LODE describing the switching system),

2) test distinguishability using conditions given in (5) which will provide the "bad" input, let us note that the second relation of (5) can be used to check if the input is a "bad" one just by checking if it satisfies the differential relation.

On line:

1) using Alien technics (see section III-A) compute $y, \dot{y}, \ldots, y^{\left(k y_{\max }\right)} ; u, \dot{u}, \ldots, u^{\left(k u_{\max }\right)}$,

${ }^{3}$ In practice at least 100 times smaller (Thus Zeno phenomenon is assume not to happen).

${ }^{4}$ This approach started in [14]. See [15] for references and [24] for interesting discussions and comparisons. 
2) check if $r_{i}(t)$ is zero for some time interval then the corresponding active subsystem is the " $i$-th",

3) deduce the continuous state estimate using 1 .

\section{Example}

Let us consider the following switching system (2) where

$$
\begin{aligned}
& i=1: \dot{x}_{1}=-x_{1}+u, y=x_{1}:(\mathrm{i} / \mathrm{o}) \dot{y}+y=u, \\
i=2: \dot{x}_{1}=x_{2}, \dot{x}_{2}=-x_{1}-x_{2}+u, y=x_{1}+x_{2}:(\mathrm{i} / \mathrm{o}) & \\
& \quad \ddot{y}+\dot{y}+y=\dot{u}+u, \\
i=3: & \dot{x}_{1}=-\frac{1}{2} x_{1}+u, y=x_{1}:(\mathrm{i} / \mathrm{o}) 2 \dot{y}+y=2 u, \\
i= & : \dot{x}_{2}=-2 x_{1}-x_{2}+u, y=x_{1}+x_{2}:(\mathrm{i} / \mathrm{o}) \ddot{y}+\dot{y}+2 y= \\
& \quad \dot{u}+u,
\end{aligned}
$$

For the first order systems, in that follows, $x_{2}$ is enforced to zero. Moreover, the output continuity is ensured between two systems whereas initial condition of derivative output is randomly chosen in $[-0.5,+0.5]$.

\begin{tabular}{|c|c|c|}
\hline$\overline{i \backslash j}$ & 1 & 2 \\
\hline 1 & $\mathrm{X}$ & $\begin{array}{l}u=u_{0} \\
y=u_{0}\end{array}$ \\
\hline 2 & $\begin{array}{l}u=u_{0} \\
y=u_{0}\end{array}$ & $x$ \\
\hline 3 & $\begin{array}{l}u=0 \\
y=0\end{array}$ & $\begin{array}{l}u=u_{0} \exp (t) \\
y=u_{0} \exp (t)\end{array}$ \\
\hline 4 & $\begin{array}{l}u=u_{0} \exp (-t) \\
y=u_{0} \exp (-t)\end{array}$ & $\begin{array}{c}u=u_{0} \exp (-t) \\
y=0\end{array}$ \\
\hline$\overline{i \backslash j}$ & 3 & $\overline{4}$ \\
\hline 1 & $\begin{array}{l}u=u_{0} \\
y=u_{0}\end{array}$ &  \\
\hline 2 & $\begin{array}{l}u=u_{0} \exp (t) \\
y=u_{0} \exp (t)\end{array}$ & $\begin{array}{c}u=u_{0} \exp (-t) \\
y=0\end{array}$ \\
\hline 3 & $\mathrm{X}$ & $\begin{array}{l}u=u_{0} \exp (3 t) \\
y=u_{0} \exp (3 t)\end{array}$ \\
\hline 4 & $\begin{array}{l}u=u_{0} \exp (3 t) \\
y=u_{0} \exp (3 t)\end{array}$ & $X$ \\
\hline
\end{tabular}

Residuals associated to previous systems are

$$
\begin{aligned}
i & =1: r_{i}=[\dot{y}]_{e}+[y]_{e}-u \\
i & =2: r_{i}=[\ddot{y}]_{e}+[\dot{y}]_{e}+[y]_{e}-[\dot{u}]_{e}-u \\
i & =3: r_{i}=2[\dot{y}]_{e}+[y]_{e}-2 u \\
i & =4: r_{i}=[\ddot{y}]_{e}+[\dot{y}]_{e}+2[y]_{e}-[\dot{u}]_{e}-u
\end{aligned}
$$

where $[\bullet]_{e}$ is the estimation of $\bullet$ and to $[y]_{e}$ corresponds the $y$ denoised signal. The following table gives the singular input/ouput for which distinuishability is lost.

Without noise, output derivatives are estimated according to the well known Euler's method. A convincing simulation when $y_{0}=u_{0}=1$ shows that systems 1 and 2 are indistinguishable (i.e. $r_{1}=r_{2}=0$ ). Figure 1 , system distinguishability is obtained easily and ensures a very good state estimation (see figure 1-(d)).

In noisy case (additive output noise $N(0,0.01)$ ), Euler's method is not available. We propose to apply recent results on derivative estimation (see [22]) in order to evaluate residuals. They are approximatively null when the associated system is active and becomes non zero in other case. However, to take the decision, that is to say to know what is the active system, is not easy (see figure 2 -(c)). Here, the mean of each residual is calculated along a sliding window. Thus at the smallest mean of residual is associated the active system. According to this logic, states are estimated (figure 2-(d)).

Figure 3, rather than to estimate output derivative in real time, a small constant and known delay is allowed for estimations (see [22] for more details). In this case, as shown figure 3-(a), in exactly the same simulation context than previously, decision according to residuals is easier. Clearly, figure 3-(b), state estimation is improved.

\section{CONCLUSION}

System distinguishability has been investigated. Easy to check necessary and sufficient conditions were obtained, which provide "bad input" that should be avoided in order to be able to distinguish the subsystems of the whole linear switching system. The proposed technics can be implemented in real time as soon as the sampling period is small enough with respect to the duration time between two successive switchings. They provide estimation of the index corresponding to the current active subsystem, and the state variable of this subsystem, via methods which are quite robust with respect to corrupting noises. Our approach will be extended to other switching systems (nonlinear, and taking into account the discrete part) as for other kind of systems (linear partial differential equations) in some future publications.

\section{REFERENCES}

[1] A.A. Agrachev, D. Liberzon, Lie-algebraic stability criteria for switched systems, SIAM J. Control Optimiz., 40:253-269, 2001.

[2] A. Alessandri, P. Coletta, Switching observers for continuous-time and discrete-time systems, Proc. ACC, pp. 25-27, Arlington, 2001.

[3] A. Arapostathis, M. Broucke, Stability and controllability of planar conwise systems, Systems Control Lett., 56:150-158, 2007.

[4] M. Babaali, G.J. Pappas, Observability of switched linear systems in continuous time, in M. Morari, L. Thiele, F. Rossi (Eds.): Proc. $8^{t h}$ Int. Workshop Hybrid Systems: Computation and Control, Lect. Notes Comput. Sci., vol. 3414, pp. 103-117, 2005.

[5] U. Boscain, Stability of planar switched systems: The linear single input case, SIAM J. Control Optimiz., 73:89-112, 2002.

[6] R. Bourdais, E. Moulay, W. Perruquetti, Stabilisation de systèmes non linéaires à commutation à l'aide de fonctions de Lyapunov contrôlées, Actes Conf. Int. Francoph. Automatique, Bordeaux, 2006.

[7] R. Bourdais, M. Fliess, C. Join, W. Perruquetti, Towards a model-free output tracking of switched nonlinear systems, Proc. NOLCOS 2007, Pretoria, pp. 637-642, 2007.

[8] M.S. Branicky, Topology of hybrid systems, Proc. $32^{\text {nd }}$ IEEE Conf. Decision Control, pp. 2309-2314, 1993.

[9] M.S. Branicky, Stability of switched and hybrid systems, Proc. $33^{\text {rd }}$ IEEE Conf. Decision Control, 1994.

[10] M.S. Branicky, Multiple Lyapunov functions and other analysis tools for switched and hybrid systems, IEEE Trans. Automat. Control, 43:475-482, 1998.

[11] R.W. Brockett, Essays on Control: Perspectives in the Theory and its Applications, chap. Hybrid models for motion control systems, pp. 29-54, Birkhäuser, 1993.

[12] J. Buisson, P.Y. Richard, H. Cormerais, On the stabilisation of switching electrical power converters, in M. Morari, L. Thiele, F. Rossi (Eds.): Proc. $8^{\text {th }}$ Int. Workshop Hybrid Systems: Computation Control, vol 3414 of Lect. Notes Comput. Sci., vol. 3414, pp. 184-197, Springer, 2005.

[13] M. Fliess. Analyse non standard du bruit. C.R. Acad. Sci. Paris, ser. I, 342:797-802, 2006.

[14] M. Fliess, C. Join, M. Mboup, and H. Sira-Ramírez. Compression différentielle de transitoires bruités. C.R. Acad. Sci. Paris,, ser. I(339):821-826, 2004.

[15] M. Fliess, C. Join, H. Sira-Ramírez. Non-linear estimation is easy, Int. J. Modelling Identification Control, 3, 2008 (available at http://hal.inria.fr/inria-00158855/en/).

[16] M. Fliess, H. Sira-Ramírez. An algebraic framework for linear identification. ESAIM Control Optim. Calc. Variat., 9:151-168, 2003. 


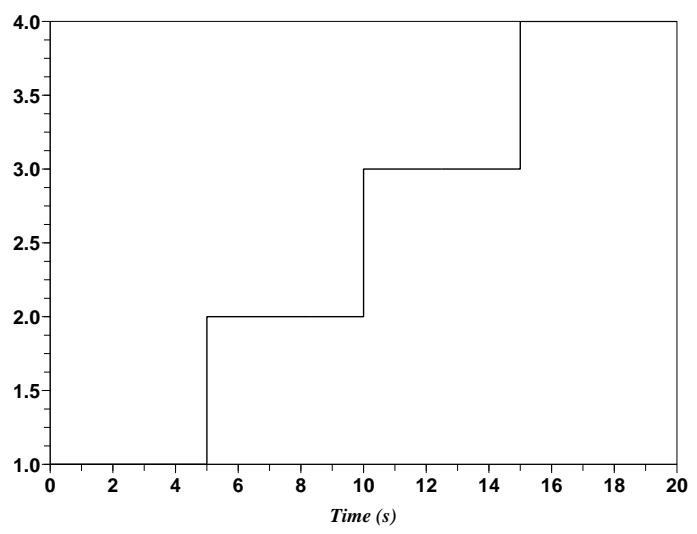

(a) Switching signal $\sigma$

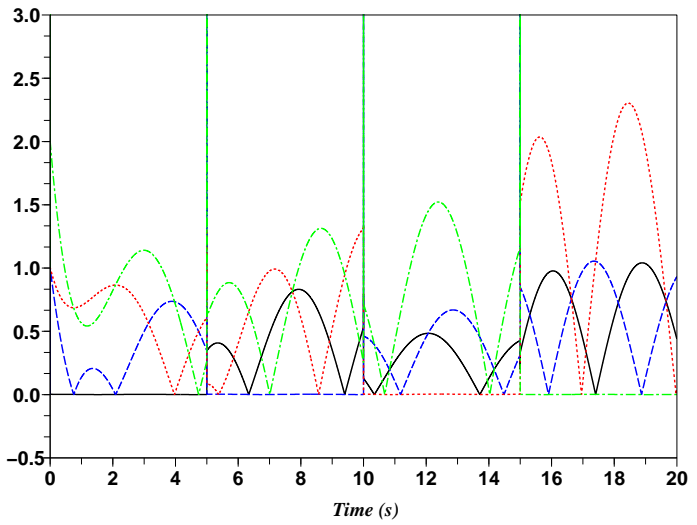

(c) Residuals : $\left|r_{1}\right|(-) ;\left|r_{2}\right|$ (- -); $\left|r_{3}\right|$ (. .); $\left|r_{4}\right|$ (- .)



(b) Output (-); input (- -)

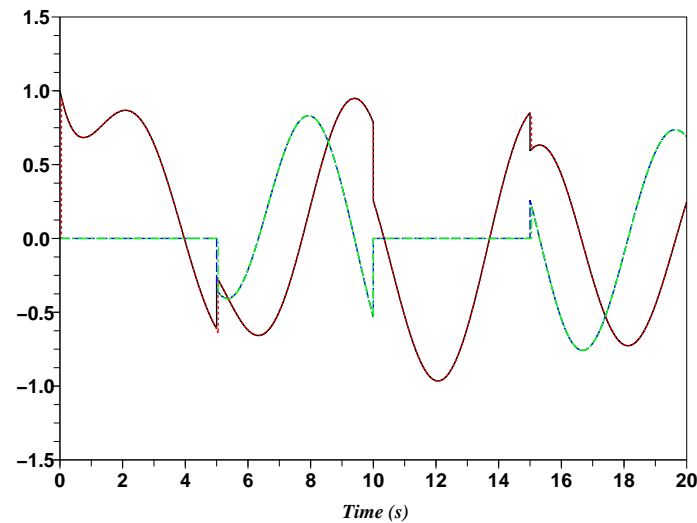

(d) State : $x_{1}(-) ; x_{2}(-) ;\left[x_{1}\right]_{e}(.) ;\left[x_{2}\right]_{e}(-$.

Fig. 1: Free noise results: sinusoidal input

[17] M. Fliess, H. Sira-Ramírez. Closed-loop parametric identification for continuous-time linear systems via new algebraic techniques In $\mathrm{H}$. Garnier, L. Wang (Eds.): Identification of Continuous-time Models from Sampled Data, pp. 363-391, Springer, 2008.

[18] J.P. Hespanha, A.S. Morse, Stability of switched systems with average dwell-time, Proc. $38^{\text {th }}$ IEEE Conf. Decision Control, pp. 2655-2660, Phoenix, 1999.

[19] D. Liberzon, Switching in Systems and Control, Boston, 2003.

[20] D. Liberzon, A.S. Morse, Basic problems in stability and design of switched systems, IEEE Control Systems Mag., 19:59-70, 1999.

[21] J.L. Mancilla-Aguilar, R.A. García, A converse Lyapunov theorem for nonlinear switched systems, Systems Control Lett., 41:67-71, 2000.

[22] M. Mboup, C. Join, M. Fliess, A revised look at numerical differentiation with an application to nonlinear feedback control, Proc. $15^{\text {th }}$ Medit. Conf. Control Automation - MED'2007, Athens, 2007 (available at http://hal.inria.fr/inria-00142588/en/).

[23] E. Moulay, R. Bourdais, W. Perruquetti, Stabilization of nonlinear switched systems using control Lyapunov functions, Nonlinear Analysis: Hybrid Systems, 1:482-490, 2007.

[24] C. Nöthen. Beiträge zur rekonstruktion nicht direkt gemessener größen bei der silizium-einkristallzüchtung nach dem czochralski-verfahren. Diplomarbeit, Technische Universität Dresden, 2007.

[25] C. De Persis, R. De Santis, A.S. Morse. Switched nonlinear systems with state dependent dwell time. Systems \& Control Letters, 50:291302, 2003

[26] C. De Persis, R. De Santis, A.S. Morse. Supervisory control with statedependent dwell-time logic and constraints. Automatica, 40:269-275, 2004.

[27] S. Pettersson. An alysis and design of hybrid systems. PhD thesis, Chalmers University of Technology, 1999.
[28] S. Pettersson and B. Lennartson. Stability and robustness for hybrid systems. In Proc. 35th IEEE Conference on Decision and Control, pages 1202-1207, 1996.

[29] E. De Santis, M.D. Di Benedetto, and G. Pola. On observability and detectability of continuous-time linear switching systems. In Proc. $42^{\text {nd }}$ IEEE Conf. Decision Control, pp. 5777-5782, Maui, Hawaii, USA, 2003.

[30] L. Schwartz. Théorie des distributions ( $2^{\text {nd }}$ ed.). Hermann, 1966.

[31] E. Skafidas, R.J. Evans, A.V. Savkin, I.R. Petersen. Stability results for switched controller systems. Automatica, 35:553-564, 1999.

[32] Z. Sun, S. Ge, T. Lee. Controllability and reachability criteria for switched linear control. Automatica, 38(5):775-786, May 2002.

[33] Z. Sun, S.S. Ge. Switched Linear Systems. Springer, 2005.

[34] R. Vidal, A. Chiuso, S. Soatto. Observability and identifiability of jump-linear systems. In Proc. of the 41st IEEE Conf. on Decision and Control, pp. 3614-3619, Las Vegas, 2002.

[35] R. Vidal, A. Chiuso, S. Soatto, S. Sastry. Observability of linear hybrid systems. In Wiedijk Freek, Maler Oded, and Pnueli Amir (Eds): Proc. $6^{\text {th }}$ Int. Workshop Hybrid Systems Computation Control - HSCC 2003, Prague, Lecture Notes in Computer Science, vol. 2623 , pp. 526-539, Springer, 2003.

[36] L. Vu, D. Liberzon. Common Lyapunov functions for families of commuting nonlinear systems. Systems Control Lett., 54:405-416, 2005.

[37] L. Wang, G. Xie, F. Hao. Linear matrix inequality approach to quadratic stabilisation of switched systems, IEE Proc. Control Theory Appl., 151: 289-294, 2004.

[38] M. Wicks and R. DeCarlo. Solution of coupled Lyapunov equations for the stabilization of multimodal linear systems. Proc. Amer. Control Conf., pp. 1709-1713, Albuquerque, 1997. 




(a) Switching signal $\sigma$

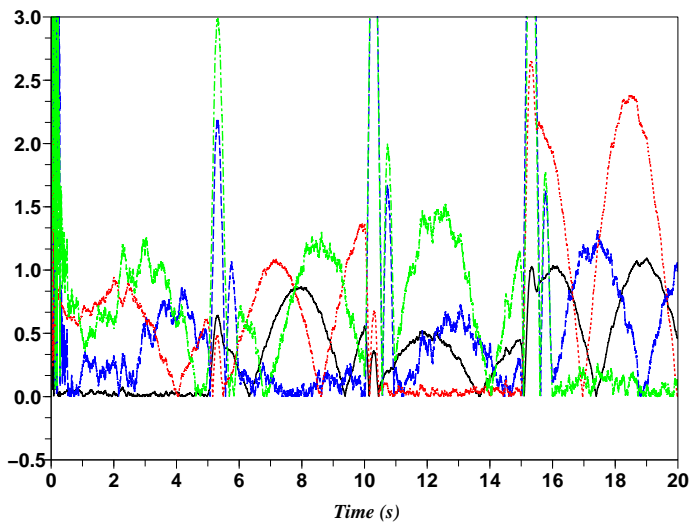

(c) Residuals : $\left|r_{1}\right|$ (-); $\left|r_{2}\right|$ (- -); $\left|r_{3}\right|$ (. .); $\left|r_{4}\right|$ (- .)

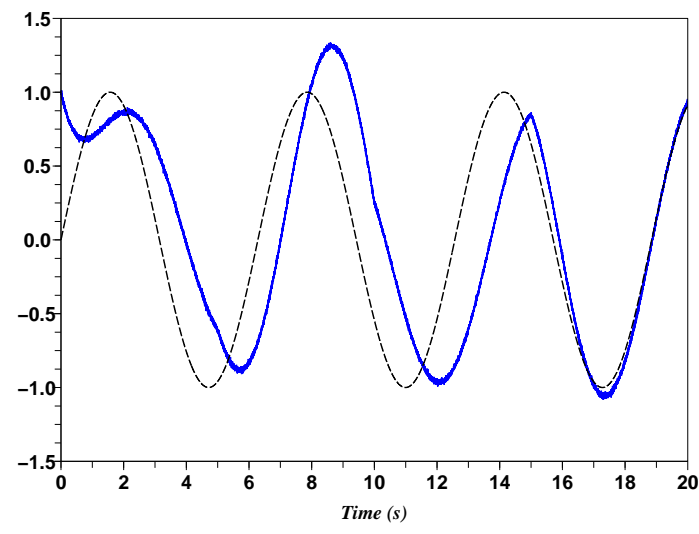

(b) Output (-); input (- -)

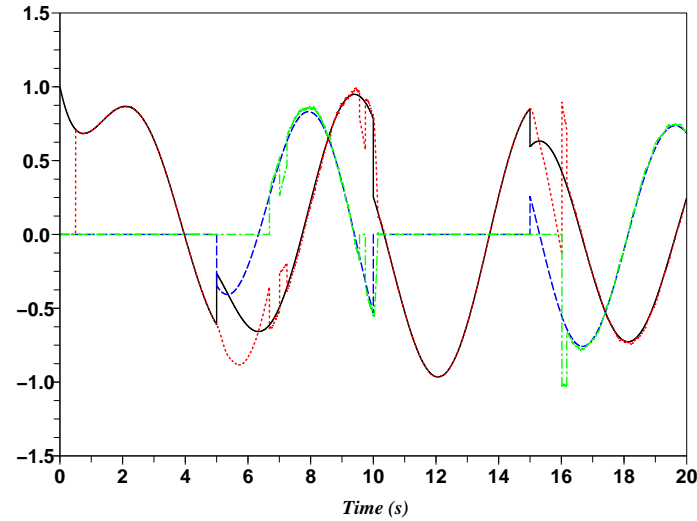

(d) State : $x_{1}(-) ; x_{2}(-) ;\left[x_{1}\right]_{e}(.) ;\left[x_{2}\right]_{e}(-$.

Fig. 2: Noised results: sinusoidal input

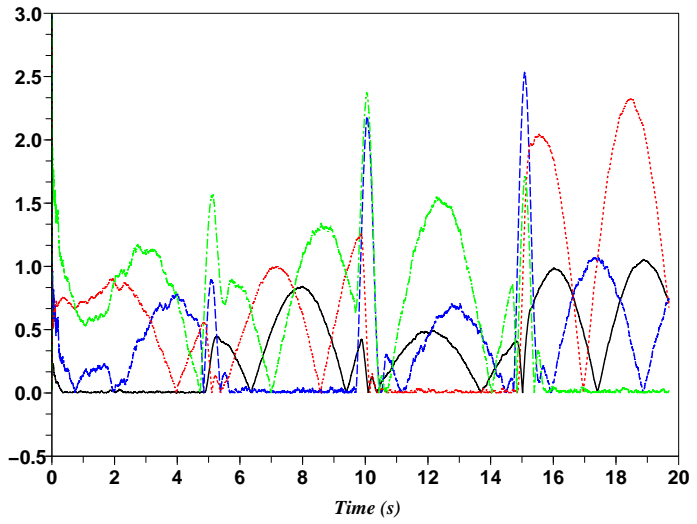

(a) Residuals : $\left|r_{1}\right|$ (-); $\left|r_{2}\right|$ (- -); $\left|r_{3}\right|$ (. .); $\left|r_{4}\right|$ (- .)



(b) State : $x_{1}(-) ; x_{2}(--) ;\left[x_{1}\right]_{e}(.) ;\left[x_{2}\right]_{e}(-$.

Fig. 3: Noised results: sinusoidal input and delayed estimations

[39] M. Wicks, P. Peleties, R. DeCarlo. Construction of piecewise Lyapunov functions for stabilizing switched systems. In Proc. 33rd IEEE Conference on Decision and Control, pp. 3492-3497, Lake Buena Vista, 1994.

[40] M. Wicks, P. Peleties, R. DeCarlo. Switched controller synthesis for the quadratic stabilization of a pair of unstable linear systems. Europ. J. Control, 4:140-147, 1998.
[41] G. Xie, D. Zheng, L. Wang. Controllability of switched linear systems. IEEE Trans. Automat. Control, 47:1401-1405, 2002.

[42] G. Zhai, H. Lin, P.J. Antsaklis. Quadratic stabilizability of switched linear systems with polytopic uncertainties. Internat. J. Control, $76: 747-753,2003$ 